\title{
Advanced risk assessment in reverse supply chain processes: A case study in Republic of Serbia
}

\author{
Aleksić, A. ${ }^{a}$, Runić Ristić, M. ${ }^{b}$, Komatina, N. ${ }^{a}{ }^{a}$, Tadić, D. ${ }^{a}$ \\ a University of Kragujevac, Faculty of Engineering, Kragujevac, Serbia \\ bUnion - Nikola Tesla University, Faculty of Management, Sremski Karlovci, Serbia
}

\begin{abstract}
A B S T R A C T
Management of a reverse supply chain (RSC) often takes place in an uncertain environment, so it is supposed to be analyzed through the proactive approach for avoidance/elimination of risks. Management initiatives based on the assessed risk level and priority of potential failure mode (PFM) should lead to the increase of business effectiveness, the competitive advantage and sustainability of the RSC. Therefore, the focus of this research is set to proposing the reliable method that would be user-friendly and suitable for the determination of risk level and priority of PFMs in RSC. Uncertainties related to the severities of Potential Effect(s) of Failure (PEF) and their frequencies', as well as detection of PFMs are described by pre-defined linguistic expressions and modelled by the interval type-2 trapezoidal fuzzy numbers (IT2TrFNs). The assessment of the relative importance of risk factors is set as a fuzzy group decision-making. The weights vector is calculated based on the procedure of fuzzy number comparison. The value of each risk factor at the level of each PFM is assessed through the predefined linguistic expressions modelled by IT2TrFNs. The rank is obtained by modified Technique for Order of Preference by Similarity to Ideal Solution (TOPSIS) method. The proposed model is tested on a real-life data from RSC that operates in Serbia. In the domain of practical implications, it may be noticed that the application of the proposed model could decrease the influence of potential causes of failures modes on the overall RSC business activities especially in the terms of strategic management and human resource practices. The novelty of the proposed model may be underlined as it is used for the analysis of different RSC activities and many interconnected issues may be solved by the proposed management measures after conducted analysis.
\end{abstract}

\author{
ARTICLE INFO \\ Keywords: \\ Reverse supply chain; \\ Risk; \\ Multi-criteria decision analysis; \\ Interval type-2 trapezoidal fuzzy \\ numbers; \\ Fuzzy FMEA framework; \\ Fuzzy TOPSIS \\ *Corresponding author: \\ nkomatina@kg.ac.rs \\ (Komatina, N.)
}

Article history:

Received 2 June 2019

Revised 28 November 2019

Accepted 2 December 2019

\section{References}

[1] Tang, O., Musa, S.N. (2011). Identifying risk issues and research advancements in supply chain risk management, International Journal of Production Economics, Vol. 133, No. 1, 25-34, doi: 10.1016/i.ijpe.2010.06.013.

[2] Tummala, V.M.R., Schoenherr, T. (2011). An implementation decision framework for supply chain management: A case study, International Journal of Logistics Systems and Management, Vol. 8, No. 2, 198-213, doi: 10.1504/ IJLSM.2011.038603.

[3] Dubey, R., Gunasekaran, A., Papadopoulos, T., Childe, S.J., Shibin, K.T., Wamba, S.F. (2017). Sustainable supply chain management: Framework and further research directions, Journal of Cleaner Production, Vol. 142, Part 2, 1119-1130, doi: 10.1016/i.jclepro.2016.03.117.

[4] Stamatis, D.H. (2003). Failure mode and effect analysis: FMEA from theory to execution, American Society for Quality, Quality Press, Milwaukee, USA. 
[5] Adhikary, D.D., Bose, G.K., Bose, D., Mitra, S. (2014). Multi criteria FMECA for coal-fired thermal power plants using COPRAS-G, International Journal of Quality \& Reliability Management, Vol. 31, No. 5, 601-614, doi: 10.1108/ IJQRM-04-2013-0068.

[6] Liu, H.-C., Liu, L., Liu, N., Mao, L.-X. (2012). Risk evaluation in failure mode and effects analysis with extended VIKOR method under fuzzy environment, Expert Systems with Applications, Vol. 39, No. 17, 12926-12934, doi: 10.1016/i.eswa.2012.05.031.

[7] Rousseau, D.M., Barends, E.G.R. (2011). Becoming an evidence-based HR practitioner, Human Resource Management Journal, Vol. 21, No. 3, 221-235, doi: 10.1111/j.1748-8583.2011.00173.x.

[8] Mendel, J.M., John, R.I., Liu, F. (2006). Interval type-2 fuzzy logic systems made simple, IEEE Transactions on Fuzzy Systems, Vol. 14, No. 6, 808-821, doi: 10.1109/TFUZZ.2006.879986.

[9] Qin, J., Liu, X. (2015). Multi-attribute group decision making using combined ranking value under interval type-2 fuzzy environment, Information Sciences, Vol. 297, 293-315, doi: 10.1016/j.ins.2014.11.022.

[10] Celik, E., Gul, M., Aydin, N., Gumus, A.T., Guneri, A.F. (2015). A comprehensive review of multi criteria decision making approaches based on interval type-2 fuzzy sets, Knowledge-Based Systems, Vol. 85, 329-341, doi: 10.1016 Li.knosys.2015.06.004.

[11] Batt, R., Colvin, A.J.S. (2011). An employment systems approach to turnover: Human resources practices, quits, dismissals, and performance, Academy of Management Journal, Vol. 54, No. 4, 695-717, doi: 10.5465/amj.2011. 64869448

[12] Liu, H.-C., Liu, L., Liu, N. (2013). Risk evaluation approaches in failure mode and effects analysis: A literature review, Expert Systems with Applications, Vol. 40, No. 2, 828-838, doi: 10.1016/i.eswa.2012.08.010.

[13] Song, W., Ming, X., Liu, H.-C. (2017). Identifying critical risk factors of sustainable supply chain management: A rough strength-relation analysis method, Journal of Cleaner Production, Vol. 143, 100-115, doi: 10.1016/i.jclepro. 2016.12.145.

[14] Chopra, S., Sodhi, M.S. (2004). Managing risk to avoid supply-chain breakdown, MIT Sloan Management Review, Vol. 46, No. 1, 53-61.

[15] Banduka, N., Tadić, D., Mačužić, I., Crnjac, M. (2018). Extended process failure mode and effect analysis (PFMEA) for the automotive industry: The FSQC-PFMEA, Advances in Production Engineering \& Management, Vol. 13, No. 2, 206-215, doi: 10.14743/apem2018.2.285.

[16] Dubois, D., Prade, H. (1998). An introduction to fuzzy systems, Clinica Chimica Acta, Vol. 270, No. 1, 3-29, doi: 10.1016/S0009-8981(97)00232-5.

[17] Zimmermann, H.-J. (2011). Fuzzy set theory - and its applications, Springer Science Business Media, New York, USA.

[18] Silva, M.M., de Gusmão, A.P.H., Poleto, T., e Silva, L.C., Costa, A.P.C.S. (2014). A multidimensional approach to information security risk management using FMEA and fuzzy theory, International Journal of Information Management, Vol. 34, No. 6, 733-740, doi: 10.1016/j.ijinfomgt.2014.07.005.

[19] Djapan, M.J., Tadic, D.P., Macuzic, I.D., Dragojovic, P.D. (2015). A new fuzzy model for determining risk level on the workplaces in manufacturing small and medium enterprises, Proceedings of the Institution of Mechanical Engineers, Part O: Journal of Risk and Reliability, Vol. 229, No. 5, 456-468, doi: 10.1177/1748006X15581219.

[20] Kahraman, C., Sarı, İ.U. (2012). Multicriteria environmental risk evaluation using type II fuzzy sets, In: Greco, S., Bouchon-Meunier, B., Coletti, G., Fedrizzi, M., Matarazzo, B., Yager, R.R. (eds), Advances in Computational Intelligence. IPMU 2012, Communications in Computer and Information Science, Springer, Berlin, Germany, 449-457, doi: $10.1007 / 978-3-642-31724-847$.

[21] Liu, H.-C., Li, P., You, J.-X., Chen, Y.-Z. (2015). A novel approach for FMEA: Combination of interval 2-tuple linguistic variables and gray relational analysis, Quality and Reliability Engineering International, Vol. 31, No. 5, 761772, doi: 10.1002 /qre.1633.

[22] Kiliç, M., Kaya, İ. (2015). Investment project evaluation by a decision making methodology based on type-2 fuzzy sets, Applied Soft Computing, Vol. 27, 399-410, doi: 10.1016/j.asoc.2014.11.028.

[23] Chen, S.-M., Lee, L.-W. (2010). Fuzzy multiple attributes group decision-making based on the interval type-2 TOPSIS method, Expert Systems with Applications, Vol. 37, No. 4, 2790-2798, doi: 10.1016/j.eswa.2009.09.012.

[24] Ghaemi Nasab, F., Rostami Malkhalifeh, M. (2010). Extension of TOPICS for group DM based on the type two fuzzy positive and negative ideal solutions, International Journal of Industrial Mathematics, Vol. 2, No. 3, $199-213$.

[25] Temur, G.T., Kaya, T., Kahraman, C. (2014). Facility location selection in reverse logistics using a type-2 fuzzy decision aid method. In: Kahraman, C., Öztayși, B. (eds), Supply Chain Management Under Fuzziness. Studies in Fuzziness and Soft Computing, Vol. 313, Springer, Berlin, Germany, 591-606, doi: 10.1007/978-3-642-53939825.

[26] Zamri, N., Abdullah, L. (2014). A new qualitative evaluation for an integrated interval type-2 fuzzy TOPSIS and MCGP, In: Herawan, T., Ghazali, R., Deris, M. (eds), Recent Advances on Soft Computing and Data Mining. Advances in Intelligent Systems and Computing, Vol. 287, Springer, Cham, Switzerland, 79-88, doi: 10.1007/978-3-31907692-8 8.

[27] Xu, Z.S. (2001). A ranking arithmetic for fuzzy mutual complementary judgment matrices, Journal of Systems Engineering, Vol. 16, No. 4, 311-314.

[28] Kahraman, C., Öztayși, B., Sarı, İ.U., Turanoğlu, E. (2014). Fuzzy analytic hierarchy process with interval type-2 fuzzy sets, Knowledge-Based Systems, Vol. 59, 48-57, doi: 10.1016/j.knosys.2014.02.001.

[29] Tang, C., Tomlin, B. (2008). The power of flexibility for mitigating supply chain risks, International Journal of Production Economics, Vol. 116, No. 1, 12-27, doi: 10.1016/i.ijpe.2008.07.008.

[30] Jharkharia, S., Shankar, R. (2007). Selection of logistics service provider: An analytic network process (ANP) approach, Omega, Vol. 35, No. 3, 274-289, doi: 10.1016/j.omega.2005.06.005. 
[31] Omega, R.S., Noel, V.M., Masbad, J.G., Ocampo, L.A. (2016). Modelling supply risks in interdependent manufacturing systems: A case study, Advances in Production Engineering \& Management, Vol. 11, No. 2, 115-125, doi: 10.14743/apem2016.2.214.

[32] Simchi-Levi, D., Wei, Y. (2012). Understanding the performance of the long chain and sparse designs in process flexibility, Operations Research, Vol. 60, No. 5, 1125-1141, doi: 10.1287/opre.1120.1081.

[33] Sharma, R.K., Kumar, D., Kumar, P. (2005). Systematic failure mode effect analysis (FMEA) using fuzzy linguistic modelling, International Journal of Quality \& Reliability Management, Vol. 22, No. 9, 986-1004, doi: 10.1108/ 02656710510625248.

[34] Kaur, A., Kanda, A., Deshmukh, S.G. (2008). Supply chain coordination: Perspectives, empirical studies and research directions, International Journal of Production Economics, Vol. 115, No. 2, 316-335, doi: 10.1016/ j.ijpe.2008.05.011.

[35] Avelar-Sosa, L., García-Alcaraz, J.L., Maldonado-Macías, A.A., Mejía-Muñoz, J.M. (2018). Application of structural equation modelling to analyse the impacts of logistics services on risk perception, agility and customer service level, Advances in Production Engineering \& Management, Vol. 13, No. 2, 179-192, doi: 10.14743/apem2018. $\underline{2.283}$. 
APEM
Advances in Production Engineering \& Management Letnik 14 | Številka 4 | December 2019 | Strani 421-434 https://doi.org/10.14743/apem2019.4.338
ISSN 1854-6250

Spletna stran: apem-journal.org Izvirni znanstveni članek

\title{
Napredna ocena tveganja pri procesih obratne dobavne verige: Študija primera v Republiki Srbiji
}

\author{
Aleksić, A. ${ }^{a}$, Runić Ristić, M. ${ }^{b}$, Komatina, N. ${ }^{a}{ }^{,}$, Tadić, D. ${ }^{a}$ \\ ${ }^{a}$ University of Kragujevac, Faculty of Engineering, Kragujevac, Serbia \\ ${ }^{b}$ Union - Nikola Tesla University, Faculty of Management, Sremski Karlovci, Serbia
}

\section{POVZETEK}

Upravljanje povratne dobavne verige (RSC) pogosto poteka $\mathrm{v}$ negotovem okolju, zato naj bi jo bilo treba analizirati s proaktivnim pristopom za preprečevanje/odpravo tveganj. Pobude upravljanja, ki temeljijo na ocenjeni stopnji tveganja in prednostnem načinu potencialnih neuspehov (PFM), bi morale voditi do povečanja poslovne učinkovitosti, konkurenčne prednosti in trajnosti RSC. Zato je poudarek te raziskave namenjen predstavitvi zanesljive metode, ki bi bila uporabniku prijazna in primerna za določanje stopnje tveganja in prioritete PFM v RSC. Negotovosti, povezane z resnostjo potencialnih učinkov neuspehov (PEF) in njihovimi frekvencami, kot tudi odkrivanje PFM-jev so opisane z vnaprej določenimi jezikovnimi izrazi in modelirane s trapeznimi mehkimi števili intervala tipa 2 (IT2TrFN). Ocenjevanje relativnega pomena dejavnikov tveganja je določeno z mehko množico sprejemanja odločitev. Vektor uteži se izračuna na podlagi postopka primerjave mehkih števil. Vrednost vsakega dejavnika tveganja na ravni vsakega PFM-ja se oceni s pomočjo vnaprej določenih jezikovnih izrazov, ki jih modelirajo IT2TrFN. Uvrstitev je pridobljena s spremenjeno tehniko za razvrstitev preferenc po podobnosti z idealno rešitvijo (TOPSIS). Predlagani model je preizkušen na resničnih podatkih RSC, ki deluje v Srbiji. Implementacija predlaganega pristopa je pokazala, da se lahko zmanjša vpliv možnih vzrokov neuspehov na celotne poslovne dejavnosti RSC, zlasti v smislu strateškega upravljanja in kadrovskih praks. Predlagan pristop se uporablja za analizo različnih dejavnosti RSC, veliko medsebojno povezanih vprašanj pa se, po izvedeni analizi, lahko reši s predlaganimi ukrepi upravljanja.

\section{PODATKI O ČLANKU}

Ključne besede:

Povratna dobavna veriga;

Tveganje;

Analiza odločitev z več kriteriji;

Trapezna mehka števila intervala

tipa-2;

Mehka metoda FMEA;

Mehka metoda TOPSIS

* Kontaktna oseba:

nkomatina@kg.ac.rs

(Komatina, N.)

Zgodovina članka:

Prejet 2. junija 2019

Popravljen 28. novembra 2019

Sprejet 2. decembra 2019 